\title{
A!
}

This is an electronic reprint of the original article.

This reprint may differ from the original in pagination and typographic detail.

Ruoho, Mikko; Valset, Kjetil; Finstad, Terje; Tittonen, Ilkka

\section{Measurement of thin film thermal conductivity using the laser flash method}

Published in:

Nanotechnology

DOI:

10.1088/0957-4484/26/19/195706

Published: 01/01/2015

Document Version

Peer reviewed version

Please cite the original version:

Ruoho, M., Valset, K., Finstad, T., \& Tittonen, I. (2015). Measurement of thin film thermal conductivity using the laser flash method. Nanotechnology, 26(19). https://doi.org/10.1088/0957-4484/26/19/195706

This material is protected by copyright and other intellectual property rights, and duplication or sale of all or part of any of the repository collections is not permitted, except that material may be duplicated by you for your research use or educational purposes in electronic or print form. You must obtain permission for any other use. Electronic or print copies may not be offered, whether for sale or otherwise to anyone who is not an authorised user. 


\title{
Measurement of thin film thermal conductivity using the laser flash method
}

\author{
Mikko Ruoho $^{1}$, Kjetil Valset ${ }^{2}$, Terje Finstad ${ }^{2}$ and Ilkka \\ Tittonen $^{1}$ \\ ${ }^{1}$ Department of Micro- and Nanosciences, Aalto University, FI-00076 Aalto, \\ Finland \\ ${ }^{2}$ Department of Physics, University of Oslo, P.O.Box 1048, Blindern, N-0316, \\ Oslo, Norway \\ E-mail: mikko.ruoho@aalto.fi
}

March 2015

\begin{abstract}
.
We present a method to measure the in-plane thermal conductivity of thin films by the laser flash technique. The method uses a well-defined structure for the analysis. We have realised the structure by conformal deposition of $\mathrm{ZnO}$ films of different thicknesses using atomic layer deposition onto a $20 \mu \mathrm{m}$ thick ion track etched polycarbonate membrane as substrate. By using this procedure we could determine the thermal conductivity of the deposited thin film from the total thermal diffusivity of the nanocomposite structures. The method has been used to obtain the in-plane thermal conductivity of the deposited $\mathrm{ZnO}$ layers within the thickness range of less than $100 \mathrm{~nm}$.
\end{abstract}

PACS numbers: 07.20.-n, 66.30.Xj, 68.60.Dv

Keywords: Thermal conductivity, Measurement, Thin film, ZnO, Laser flash analysis, atomic layer deposition, nanofabrication

The presence of nanostructures such as thin films and nanowires modify the thermal conductivity since structures at the nanoscale limit the wavelengths of the phonon spectrum in a material. Moreover, nanostructures play an important role in many devices giving rise to the increasing interest in the thermal conduction in nanostructures. The ability to tune thermal conduction is beneficial in the thermal management of electronic and mechanical components. For the special case of thermoelectrics, a reduction of the thermal conductivity without drastically lowering electrical conductivity is needed for efficient thermoelectric energy conversion.

The most commonly utilised methods to measure thermal conductivity of thin films include the $3 \omega$ method [1] and time-domain thermoreflectance [2]. The $3 \omega$ method utilises a thin conductor wire fabricated onto the sample of interest. Since this conducting wire functions both as a heater as well as a thermometer, the dimensions as well as the thermal properties of the conductor need to be known very precisely. In addition, the conductor should be electrically insulated from the sample being measured. Having met these requirements, the method is very versatile, and enables measurements of thermal conductivity both in-plane and out-of-plane directions as well as measurements of very thin samples. $[3,4]$ Nevertheless, due to the experimental 
impracticality of the $3 \omega$ method, the time-domain thermoreflectance has recently become more popular [5]. In a time-domain thermoreflectance scheme a sample is coated with a metal film which is used as an absorber for the heating laser as well as a reflector for the thermal measurement. Similarly to the $3 \omega$ method, the thermal and geometrical properties of the transducer need to be known as the metal film is used to probe the thermal response of the sample to a transient heat pulse. Thermometry is accomplished by detecting changes in reflectance of the transducer film and the determination of the thermal properties of the sample are accomplished by adjusting free parameters in a phenomenological model. The method allows the determination of out-of-plane thermal diffusivity of bulk material, thin films and interfaces. In addition, the method allows spatial mapping of these properties. However, the method has its major limitations in the in-plane measurement of thermal conductivity as the method is mostly sensitive in the out-of-plane direction. Currently, measurements of in-plane directions are only possible for highly conductive films on insulating substrates $[5,6]$.

The laser flash method [7] is commonly used method for measurement of the thermal properties of macroscopic samples. The samples are usually prepared for the method by spraying a layer of graphite on the samples to act as an absorber for the heating laser on one side and as an emitter for the IR detector on the other. The measurement is done by heating one side of the sample by a short pulse of energy and detecting the corresponding temperature rise from the back side of the sample. The thermal properties can be calculated from the measured temperature rise as a function of time. The method allows fast measurements with accurate and reproducible results. However, the thickness of the sample that can be measured is limited by the timescales associated with heat pulse and detection. With typical commercial instruments these factors correspond to minimum sample thicknesses of $10-100 \mu \mathrm{m}$ depending on the thermal conductivity of the sample.

While atomic layer deposition (ALD) has often been used to deposit structures on porous polymer substrates [8], their thermal properties are a lot less studied. The unique properties of ALD method are the highly conformal growth [9] and precise thickness control due to the self-limiting growth mechanism. Hence, the method allows large scale fabrication of complex geometries, making it of particular interest for the production of thermoelectric nanostructures.

The laser flash method has been previously utilised to study thermal properties of nickel [10], silver [11] and silicon [12] nanowires. While these reports present an estimation for the thermal conductivity of the given materials under study, the inability to control the thickness of the structure during the deposition as well as uncertainties in pore filling make the methods impractical and inaccurate for probing the properties of the deposited film itself.

Here we present a way to use established laser flash equipment to measure the thermal diffusivity of nanocomposites of ALD deposited thin film structure and tracketched polycarbonate membrane. In addition, we use the data to calculate the thermal conductivity of the deposited thin films. The thermal properties of ALD deposited $\mathrm{ZnO}$ are of interest due to their thermoelectric properties [13]. The work allows for the widely used laser flash measurement method to be extended for temperature dependent determination of thermal properties of thin films. 
Measurement of thin film thermal conductivity using the laser flash method

Table 1. Deposition parameters and resulting total thin film thicknesses of the samples. The sample 6 is polycarbonate substrate without a thin film.

\begin{tabular}{lcll}
\hline $\begin{array}{l}\text { Name } \\
\text { of sample }\end{array}$ & $\begin{array}{l}\text { Thin film } \\
\text { thickness (nm) }\end{array}$ & $\begin{array}{l}\text { Number of } \\
\text { DEZn cycles }\end{array}$ & $\begin{array}{l}\text { Number of } \\
\text { TMA cycles }\end{array}$ \\
\hline Sample 1 & 102 & 400 & 80 \\
Sample 2 & 59 & 200 & 80 \\
Sample 3 & 35 & 100 & 80 \\
Sample 4 & 17 & 25 & 80 \\
Sample 5 & 12 & 0 & 80 \\
Sample 6 & 0 & 0 & 0 \\
\hline
\end{tabular}

\section{Materials and methods}

\subsection{Sample preparation, deposition and characterisation}

Atomic layer deposition was used to grow $\mathrm{Al}_{2} \mathrm{O}_{3}$ and $\mathrm{ZnO}$ nanolaminates on tracketched polycarbonate (PC) membranes (Cyclopore, GE Healthcare). The substrates were measured to have an average pore diameter of $955 \mathrm{~nm}$ and density of $2.2 \times 10^{7}$ pores $\mathrm{cm}^{-2}$. With the substrate thickness of $20 \mu \mathrm{m}$ the pores of the substrate had an aspect ratio of about 21. BENEQ TFS-500 ALD reactor was used and ALD growth of $\mathrm{ZnO}$ was done by sequential pulsing of diethylzinc (DEZn) and $\mathrm{H}_{2} \mathrm{O}$. The growth of $\mathrm{Al}_{2} \mathrm{O}_{3}$ was carried out by pulsing of trimethylaluminium (TMA) and $\mathrm{H}_{2} \mathrm{O}$. The deposition was performed at $130{ }^{\circ} \mathrm{C}$ with long total pulse lengths in order to achieve uniform growth within the pores. The total pulse lengths were $900 \mathrm{~ms}$ for TMA, 750 ms for DEZn and $750 \mathrm{~ms}$ for $\mathrm{H}_{2} \mathrm{O}$.

The deposition consisted of 40 TMA and $\mathrm{H}_{2} \mathrm{O}$ pulses, followed by a varying amount $\left(25,100,200\right.$ and 400) of DEZn and $\mathrm{H}_{2} \mathrm{O}$ pulses. The deposition was finished with pulsing of additional 40 cycles of TMA and $\mathrm{H}_{2} \mathrm{O}$. The samples and their thicknesses are shown in table 1. The deposition of an initial $\mathrm{Al}_{2} \mathrm{O}_{3}$ layer on the polycarbonate was found to be beneficial for the nucleation of the succeeding $\mathrm{ZnO}$ layer

A Ti/Au bi-layer layer of $20 \mathrm{~nm}$ of each element was deposited on both sides of the samples by e-beam evaporation in order to minimise radiative heat transport through the translucent substrate during the measurement. The samples were also annealed at $120^{\circ} \mathrm{C}$ for 19 hours. A graphite layer (Graphit 33, Kontakt Chemie) was sprayed on the samples as is typically done with laser flash analysis (LFA) sample preparation. However, as the substrates are thin, the thickness of the graphite layer may contribute a significant portion of the total thickness. Thus, we measured the thicknesses of each sample using Hitachi TM3000 scanning electron microscope (SEM). In addition, Energy dispersive x-ray (EDX) elemental analysis was obtained with Bruker Quantax 70. The high resolution SEM imaging was done with Zeiss Supra 40.

The thicknesses of the films were measured by Plasmos SD 2300 ellipsometer using samples grown on silicon (100) in the same batch as the nanocomposites. In addition, the thicknesses of the thin films deposited on polymer were evaluated from SEM images complementing the results obtained with ellipsometer. Nevertheless, we note that the nucleation of the films is dependent on the substrate and might cause an error in the absolute value of the film thickness. The films could be somewhat thicker on the polymer substrate than on silicon. [14] Nevertheless, since the growth is similar 

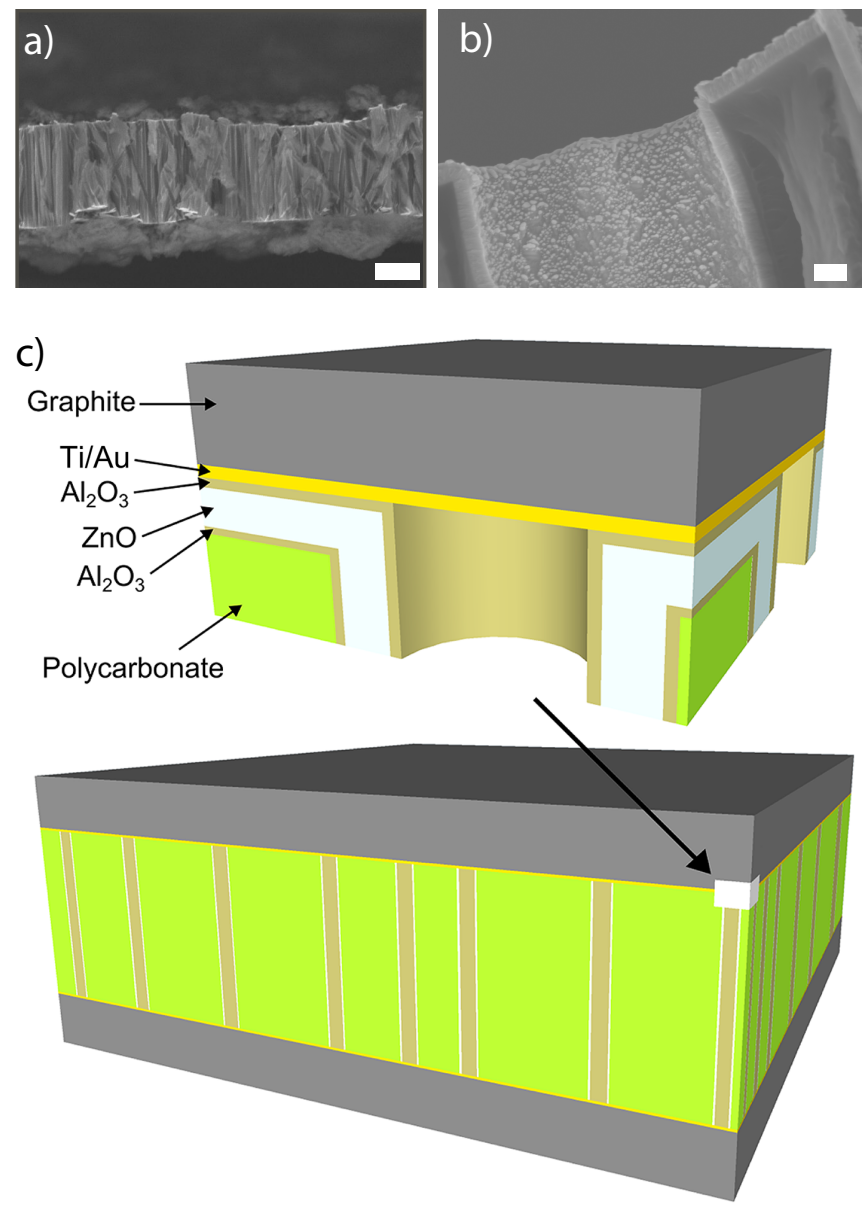

Figure 1. The structure of the nanocomposite: a) fractured surface of the nanocomposite array including graphite layers on both sides of the structure b) fractured surface of the nanocomposite array without graphite layers c) schematic representation of the structure. The scale bars are a) $10 \mu \mathrm{m}$ and b) $200 \mathrm{~nm}$.

on both silicon and polymers after an initial nucleation [14], the phenomenon does not contribute to the difference of the film thicknesses between the samples which is used to obtain the thermal conductivity.

\subsection{Thermal conductivity measurement procedure}

The laser flash thermal diffusivity measurements were carried out with Netzsch LFA 457. The measured data were analysed using a three-layer-model in order to take into account the possible contribution of the graphite layers.

The thermal conductivity of the deposited thin film can be obtained by plotting the thermal properties of the nanocomposites as a function of the relative area of the thin film of interest.

A simple model utilising modified effective medium theory [15] may be used to justify the analysis of the measurement results. The effective thermal conductivity 
for a composite material with aligned continuous fibres perpendicular to the sample surface is

$$
\kappa_{\text {meas }}=\kappa_{\text {film }} \phi+\kappa_{\text {sub }}(1-\phi),
$$

where $\phi$ is the volume percentage of the deposited thin film, $\kappa_{f i l m}$ and $\kappa_{\text {sub }}$ are the thermal conductivities of the thin film and the substrate, respectively. By replacing

$$
\phi=\frac{V_{\text {film }}}{V_{\text {sub }}+V_{\text {film }}}=\frac{A_{\text {film }}}{A_{\text {sub }}+A_{\text {film }}}
$$

and rearranging the equation, leads to

$$
\kappa_{\text {meas }} \frac{A_{\text {film }}+A_{\text {sub }}}{A_{\text {sub }}}=\kappa_{\text {film }} \frac{A_{\text {film }}}{A_{\text {sub }}}+\kappa_{\text {sub }},
$$

where $\mathrm{V}_{\text {film }}$ and $\mathrm{V}_{\text {sub }}$ are the volumes and $\mathrm{A}_{\text {film }}$ and $\mathrm{A}_{\text {sub }}$ are the cross sectional areas of the thin film and substrate, respectively. Reformulation of the left-hand side of the equation with

$$
\kappa=\alpha \rho c
$$

is needed since the parameter obtained directly by the laser flash measurement is the thermal diffusivity, $\alpha$. Also, the specific heat capacity, $c$, and the density, $\rho$, are introduced to the expressions. The equation becomes

$$
\alpha_{\text {meas }} \rho_{\text {meas }} c_{\text {meas }} \frac{A_{\text {film }}+A_{\text {sub }}}{A_{\text {sub }}}=\kappa_{\text {film }} \frac{A_{\text {film }}}{A_{\text {sub }}}+\kappa_{\text {sub }},
$$

where the volumetric heat capacity of the sample is

$$
\rho_{\text {meas }} c_{\text {meas }}=\frac{V_{\text {film }}}{V_{\text {film }}+V_{\text {sub }}} \rho_{\text {film }} c_{\text {film }}+\frac{V_{\text {sub }}}{V_{\text {film }}+V_{\text {sub }}} \rho_{\text {sub }} c_{\text {sub }} .
$$

By combining equations 5 and 6 we get the exact analytical expression for handling the measurement results and determination of the film properties

$$
\alpha_{\text {meas }}\left(\rho_{\text {film }} c_{\text {film }} \frac{A_{\text {film }}}{A_{\text {sub }}}+\rho_{\text {sub }} c_{\text {sub }}\right)=\kappa_{\text {film }} \frac{A_{\text {film }}}{A_{\text {sub }}}+\kappa_{\text {sub }} .
$$

Hence, by fitting a line to $\kappa_{\text {meas }} \frac{A_{\text {film }}+A_{\text {sub }}}{A_{\text {sub }}}$ as a function of $\frac{A_{\text {film }}}{A_{\text {sub }}}$, we can obtain the thermal conductivities of both the thin film and the substrate.

The procedure presented in equation (7) includes an assumption of constant thermal conductivity. In a case of thickness dependent thermal conductivity, the fitting should be carried out in such a range of $\frac{A_{\text {film }}}{A_{\text {sub }}}$ in which the thermal conductivity may be considered constant.

It might be worth noting that in order to analyse the results in this way, there is no requirement that the deposited film thickness needs to be uniform. However, the thickness variation should be known and for this reason it is very convenient to have uniform film thickness for the determination of the conduction area of the deposited film.

Values used for the calculations were taken from [16-19] and the density of the polycarbonate substrate was measured. The values used for each constituent were $\rho_{Z n O}=5.62 \mathrm{~g} \mathrm{~cm}^{-3}, \rho_{\mathrm{Al}_{2} O_{3}}=3.15 \mathrm{~g} \mathrm{~cm}^{-3}, \rho_{P C}=1.12 \mathrm{~g} \mathrm{~cm}^{-3}, c_{Z n O}=0.46$ $\mathrm{Jg}^{-1} \mathrm{~K}^{-1}, c_{\mathrm{Al}_{2} \mathrm{O}_{3}}=0.79 \mathrm{Jg}^{-1} \mathrm{~K}^{-1}$ and $c_{P C}=1.2 \mathrm{Jg}^{-1} \mathrm{~K}^{-1}$.

The results are obtained for $\mathrm{ZnO}$ alone since all the samples have the same interfaces and the only variable between the different samples is the $\mathrm{ZnO}$ film thickness. The contribution of potential thermal boundary resistances at the interfaces of the 


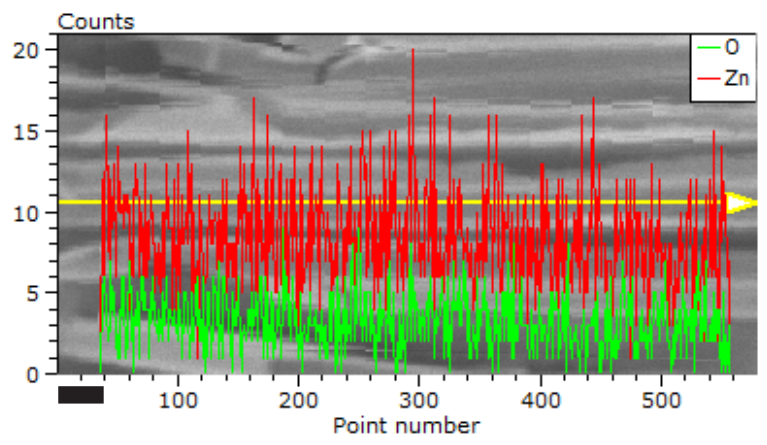

Figure 2. EDX line scan from the middle of a fractured nanocomposite (Sample 1) showing the relative x-ray intensities of $Z_{n} L_{\alpha}$ and $O K_{\alpha}$. The background is a SEM image and the yellow arrow shows the location of the line scan which is oriented along the direction of a fractured pore. The scale bar is $1 \mu \mathrm{m}$.

different material layers could also play a role in the measurement if they are not identical in each sample. Hence, the approach has similarities with the handling of thermal boundary resistances in differential $3 \omega$ method [3,20].

Reproducibility and repeatability of the measured results were verified by measuring samples prepared separately from the same batch (i.e. substrate). The obtained values from single flashes agree within $8 \%$ and the average values at given temperature within $4 \%$. In addition, the possible effect of the graphite layer thicknesses was studied by preparing a pair of samples with different graphite layer thicknesses. The obtained diffusivity values for single flashes agree within $7 \%$ and the average values for a given temperature within $4 \%$. Although, the thickness of the graphite layers may be considerable, it does not contribute to the total thermal resistance since it has two orders of magnitude higher thermal conductivity than polycarbonate. Therefore, it plays no significant role in the measurement results.

\section{Results and discussion}

\subsection{Structural properties}

The average pore distribution and diameter of the pores in the substrate play a role in the calculation of the area. Hence they are measured from SEM images. The assumptions in the measurement are that the film thickness inside the pores is uniform and that the pores are perpendicular to the surface of the substrate. Figure 2 shows the relative distributions of $\mathrm{Zn}$ and $\mathrm{O}$ along the length of a single nanotube justifying the assumption of the uniformity of the deposited film within the pores.

\subsection{Thermal conductivity measurements}

Figure 3 shows the temperature dependent total thermal diffusivity of the nanocomposite samples and references. The data shows temperature independent thermal diffusivity in the measured range. The thermal diffusivity of $0.078 \mathrm{~mm}^{2} \mathrm{~s}^{-1}$ of the polycarbonate substrate was increased to $0.17 \mathrm{~mm}^{2} \mathrm{~s}^{-1}$ for the sample with the 


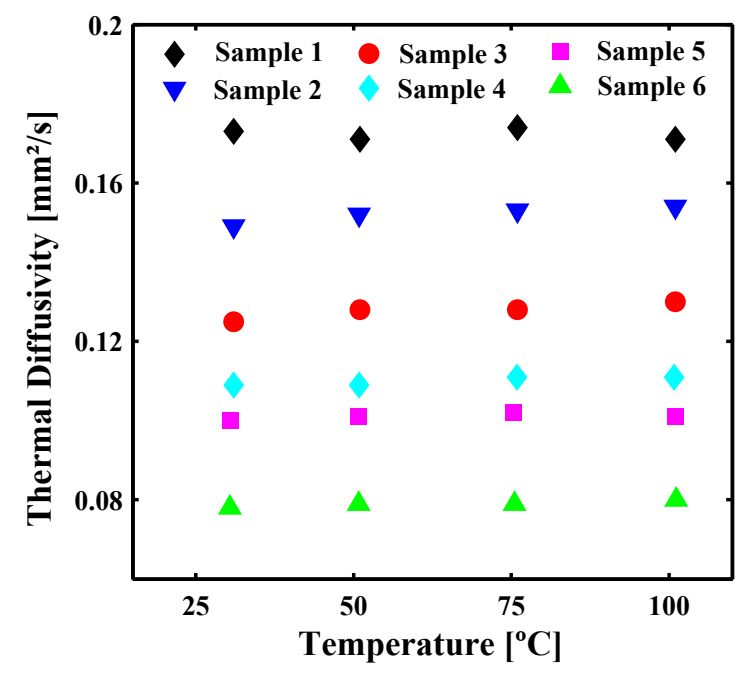

Figure 3. Temperature dependent thermal diffusivities of the nanocomposites as well as the polycarbonate substrate

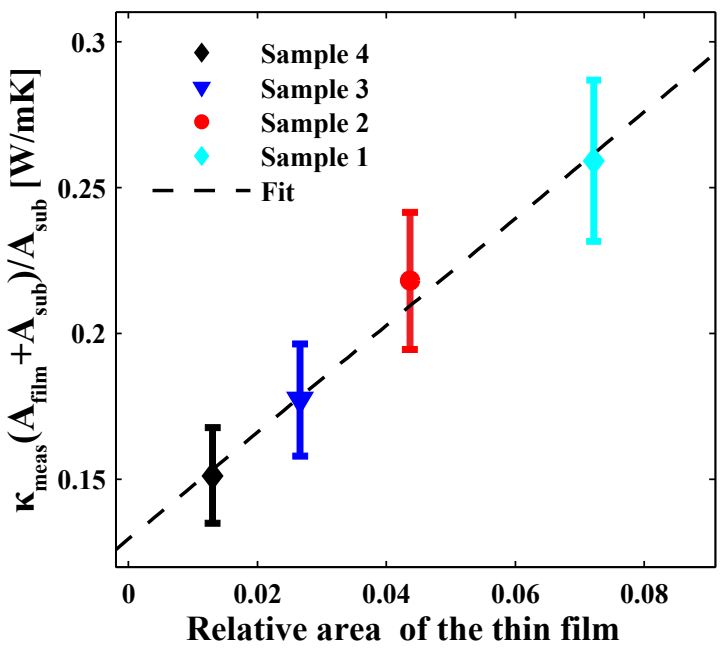

Figure 4. $\kappa_{\text {meas }} \frac{A_{\text {film }}+A_{s u b}}{A_{s u b}}$ of the nanocomposite samples as a function of $\frac{A_{\text {film }}}{A_{\text {substrate }}}$. Dashed line represents the linear least squares fit to the values.

thickest thin film. Additionally, the difference in the thermal diffusivity between the samples is independent of the temperature.

Figure 4 shows the determination of the thin film thermal conductivity according to equation (7). The y-axis values were obtained using the thermal diffusivity values shown in figure 3 and transforming them to thermal conductivity values with equations (4) and (6). The obtained thermal conductivity values were then multiplied with $\frac{A_{\text {film }}+A_{\text {sub }}}{A_{\text {sub }}}$. When plotting the values as a function of $\frac{A_{\text {film }}}{A_{\text {subs }}}$, the fit yields the 
thermal conductivity of the thin film. A value of $1.9 \pm 0.2 \mathrm{~W} \mathrm{~m}^{-1} \mathrm{~K}^{-1}$ was obtained at $30^{\circ} \mathrm{C}$. The linear dependence of the thermal conductivity on both the deposited film area and thickness suggest a thickness independent thermal conductivity. Within the measured temperature range of $30-100{ }^{\circ} \mathrm{C}$, the thermal conductivity of $\mathrm{ZnO}$ thin film indicates a slight increase up to $2.1 \mathrm{~W} \mathrm{~m}^{-1} \mathrm{~K}^{-1}$. The thermal conductivity of the substrate, however, increases from 0.13 to $0.17 \mathrm{~W} \mathrm{~m}^{-1} \mathrm{~K}^{-1}$ within the temperature range. The increase is likely due to the increasing temperature dependencies of thermal conductivity for both constituents of the substrate, $\mathrm{PC}$ and $\mathrm{Al}_{2} \mathrm{O}_{3}$.

The thermal conductivity of the substrate was obtained from plotting the results of samples $1-4$ as illustrated in figure 4 . However, the values may also be obtained from the measurements of sample 5 , shown in figure 3 . The results obtained with different methods agree within $2-6 \%$ for the different temperature points of measurement. Hence, there is no need for separate measurement of the thermal diffusivity of the substrate.

The measured thermal conductivity for $\mathrm{ZnO}$ thin film of about $1.9 \mathrm{~W} \mathrm{~m}^{-1} \mathrm{~K}^{-1}$ ranks within the lower range which is typically reported for $\mathrm{ZnO}$ thin films as values ranging from 1 to $40 \mathrm{~W} \mathrm{~m}^{-1} \mathrm{~K}^{-1}$ have been published [21-26]. Nevertheless, some

data for in-plane thermal conductivity has been published only by Xu et. al. [25], who reported a value of $2.6 \mathrm{~W} \mathrm{~m}^{-1} \mathrm{~K}^{-1}$.

\subsection{Experimental uncertainties}

The largest contribution to the measurement uncertainty arises from the LFA measurement including corrections for the heat loss and finite heating pulse. The uncertainty for a single measurement is estimated to be $8.2 \%$. In addition, the values used for specific heat constitute a considerable source of uncertainty, which is estimated to be $5 \%$. The density of the material can be determined more accurately and the uncertainty is estimated to be $2 \%$. Also, the thin film thickness of the samples gives rise to an uncertainty of $0.5-4.4 \%$ depending on the film thickness, thinner films giving higher uncertainty. Hence, the combined standard uncertainty for the least squares fit to obtain the thermal conductivity is $10-13 \%$ depending on the linearity of the measured data at each temperature as well as number of repeated measurements.

\section{Conclusions}

A well-defined nanocomposite is fabricated by conformal deposition of a thin film structure on a track-etched polycarbonate substrate. The ability to tune the thickness of the deposited film in a precise manner allows tuning of corresponding total thermal conductivity of the nanocomposite. By combining the information from different structures, the thermal conductivity of the thin film can be extracted.

The presented procedure offers a possibility for determination of in-plane thermal conductivity of thin film samples. The procedure allows experimentally convenient way of studying thermal behaviour of thin films having a thickness that is less than $100 \mathrm{~nm}$. Hence, the method widens the film thickness range that may be studied with standard LFA equipment by more than two orders of magnitude. The method is best suited for materials which can be deposited in a conformal fashion. Hence, it opens new possibilities in the study of atomic layer deposited films as well as potentially for other chemical vapour deposition methods. 


\section{Acknowledgments}

We thank A. Perros for advices on fabricational aspects. MR and IT gratefully acknowledge the financial support from the Academy of Finland project number 13140009, Aalto ELEC Doctoral School and Energy Efficiency Programme (project 9158101). TF and KV acknowledge support from the Norwegian Research Council under Contract NFR11-40-6321 (NanoThermo), and the University of Oslo. This research was partly performed at the Micronova Nanofabrication Centre, supported by Aalto University.

[1] David G. Cahill. Thermal conductivity measurement from 30 to $750 \mathrm{k}$ : the 3 method. Review of Scientific Instruments, 61(2), 1990.

[2] Carolyn A. Paddock and Gary L. Eesley. Transient thermoreflectance from thin metal films. Journal of Applied Physics, 60(1), 1986.

[3] T. Borca-Tasciuc, A. R. Kumar, and G. Chen. Data reduction in 3 method for thin-film thermal conductivity determination. Review of Scientific Instruments, 72(4):2139-2147, 2001.

[4] J. Kimling, S. Martens, and K. Nielsch. Thermal conductivity measurements using 1 and 3 methods revisited for voltage-driven setups. Review of Scientific Instruments, 82(7):-, 2011.

[5] David G. Cahill, Paul V. Braun, Gang Chen, David R. Clarke, Shanhui Fan, Kenneth E. Goodson, Pawel Keblinski, William P. King, Gerald D. Mahan, Arun Majumdar, Humphrey J. Maris, Simon R. Phillpot, Eric Pop, and Li Shi. Nanoscale thermal transport. ii. 2003-2012. Applied Physics Reviews, 1(1):-, 2014.

[6] Joseph P. Feser and David G. Cahill. Probing anisotropic heat transport using time-domain thermoreflectance with offset laser spots. Review of Scientific Instruments, 83(10):-, 2012.

[7] W. J. Parker, R. J. Jenkins, C. P. Butler, and G. L. Abbott. Flash method of determining thermal diffusivity, heat capacity, and thermal conductivity. Journal of Applied Physics, 32(9), 1961.

[8] H. Shin, D.-K. Jeong, J. Lee, M.M. Sung, and J. Kim. Formation of tio2 and zro2 nanotubes using atomic layer deposition with ultraprecise control of the wall thickness. Advanced Materials, 16(14):1197-1200, 2004.

[9] Mikko Ritala, Markku Leskel, Jan-Pieter Dekker, Cees Mutsaers, Pekka J. Soininen, and Jarmo Skarp. Perfectly conformal tin and al2o3 films deposited by atomic layer deposition. Chemical Vapor Deposition, 5(1):7-9, 1999.

[10] Kafil M. Razeeb and Saibal Roy. Thermal diffusivity of nonfractal and fractal nickel nanowires. Journal of Applied Physics, 103(8):-, 2008.

[11] Ju Xu, Alessio Munari, Eric Dalton, Alan Mathewson, and Kafil M. Razeeb. Silver nanowire array-polymer composite as thermal interface material. Journal of Applied Physics, 106(12):-, 2009.

[12] Ting Zhang, Shao long Wu, Rui ting Zheng, and Guo an Cheng. Significant reduction of thermal conductivity in silicon nanowire arrays. Nanotechnology, 24(50):505718, 2013.

[13] Mikko Ruoho, Ville Pale, Mikhail Erdmanis, and Ilkka Tittonen. Influence of aluminium doping on thermoelectric performance of atomic layer deposited zno thin films. Applied Physics Letters, 103(20):-, 2013.

[14] C. A. Wilson, R. K. Grubbs, and S. M. George. Nucleation and growth during al2o3 atomic layer deposition on polymers. Chemistry of Materials, 17(23):5625-5634, 2005.

[15] Ce-Wen Nan, R. Birringer, David R. Clarke, and H. Gleiter. Effective thermal conductivity of particulate composites with interfacial thermal resistance. Journal of Applied Physics, 81(10), 1997.

[16] J. M. Jensen, A. B. Oelkers, R. Toivola, D. C. Johnson, J. W. Elam, and S. M. George. Xray reflectivity characterization of zno/al2o3 multilayers prepared by atomic layer deposition. Chemistry of Materials, 14(5):2276-2282, 2002.

[17] Jun Liu, Byunghoon Yoon, Eli Kuhlmann, Miao Tian, Jie Zhu, Steven M. George, Yung-Cheng Lee, and Ronggui Yang. Ultralow thermal conductivity of atomic/molecular layer-deposited hybrid organicinorganic zincone thin films. Nano Letters, 13(11):5594-5599, 2013.

[18] Umesh Gaur, Sukfai Lau, and Bernhard Wunderlich. Heat capacity and other thermodynamic properties of linear macromolecules. ix. final group of aromatic and inorganic polymers. Journal of Physical and Chemical Reference Data, 12(1), 1983.

[19] YS Touloukian and EH Buyco. Thermophysical Properties of Matter Specific Heat Nonmetallic Solids, volume 5. IFI/Plenum, New York, 1970. 
[20] D.W. Song, C. Caylor, W.L. Liu, T. Zeng, T. Borca-Tasciuc, T.D. Sands, and G. Chen. Thermal conductivity characterization of skutterudite thin films. In Thermoelectrics, 1999. Eighteenth International Conference on, pages 679-682, Aug 1999.

[21] Joana Loureiro, Nuno Neves, Raquel Barros, Tiago Mateus, Rafael Santos, Sergej Filonovich, Sebastian Reparaz, Clivia M. Sotomayor-Torres, Frederic Wyczisk, Laurent Divay, Rodrigo Martins, and Isabel Ferreira. Transparent aluminium zinc oxide thin films with enhanced thermoelectric properties. J. Mater. Chem. A, 2:6649-6655, 2014.

[22] J. Alvarez-Quintana, E. Martnez, E. Prez-Tijerina, S. A. Prez-Garca, and J. Rodrguez-Viejo. Temperature dependent thermal conductivity of polycrystalline zno films. Journal of Applied Physics, 107(6):-, 2010.

[23] Nina Vogel-Schäuble, Tino Jaeger, Yaroslav E. Romanyuk, Sascha Populoh, Christian Mix, Gerhard Jakob, and Anke Weidenkaff. Thermal conductivity of thermoelectric al-substituted zno thin films. physica status solidi (RRL) Rapid Research Letters, 7(5):364-367, 2013.

[24] Shrikant Saini, Paolo Mele, Hiroaki Honda, Dave J. Henry, Patrick E. Hopkins, Leopoldo MolinaLuna, Kaname Matsumoto, Koji Miyazaki, and Ataru Ichinose. Enhanced thermoelectric performance of al-doped zno thin films on amorphous substrate. Japanese Journal of Applied Physics, 53(6):060306, 2014.

[25] Yibin Xu, Masahiro Goto, Ryozo Kato, Yoshihisa Tanaka, and Yutaka Kagawa. Thermal conductivity of zno thin film produced by reactive sputtering. Journal of Applied Physics, 111(8):-, 2012.

[26] Zheng Xing Huang, Zhen An Tang, Jun Yu, and Suyuan Bai. Thermal conductivity of nanoscale polycrystalline zno thin films. Physica B: Condensed Matter, 406(4):818 - 823, 2011. 